\title{
FACTORES SOCIOECONÓMICOS DEL HOGAR EN LA ELECCIÓN DEL TIPO DE GESTIÓN DEL ESTABLECIMIENTO EDUCATIVO EN ARGENTINA
}

\author{
Household Socioeconomic Characteristics \\ on School Regime Choices in Argentina
}

\author{
Juan Segnana ${ }^{1}$ \\ ORCID: 0000-0002-4283-7883 \\ Cecilia Adrogué ${ }^{2}$ \\ ORCID: 0000-0002-3716-7352
}

\begin{abstract}
${ }^{1}$ Centro de Estudios para el Desarrollo Humano. Universidad de San Andrés, Argentina.
${ }^{2}$ Consejo Nacional de Investigaciones Científicas y Técnicas. Universidad de San Andrés, Argentina.

Correos: juansegnana@gmail.com; cadrogue@gmail.com
\end{abstract}

Recibido: 22/02/2021

Aceptado: $13 / 05 / 2021$

Resumen: En los últimos años se observa un incremento en la proporción de alumnos en instituciones de gestión privada en la educación básica Argentina, inclusive entre los hogares de menor nivel socioeconómico. Esto pone en agenda el tema de la segregación educativa y de cuáles son los factores que inciden en ella. En este trabajo se analiza la influencia de los factores socioeconómicos del hogar en la probabilidad de que los hijos asistan a un establecimiento de gestión pública o privada tanto de nivel primario como secundario en Argentina. También se muestra la composición del alumnado de nivel primario y secundario según el nivel educativo del jefe de hogar y la clase social a la que ese hogar pertenece haciendo uso de los datos de la Encuesta Permanente de Hogares para Argentina. Los resultados van en línea con hallazgos complementarios de la literatura de educación.

Palabras clave: probabilidad; gestión escolar; educación primaria; educación secundaria; estratificación socioeconómica; Argentina.

\begin{abstract}
The proportion of students enrolled in private schools has been increasing during the past years in Argentina, even for households with fewer socioeconomic resources. Thus, the study of educational segregation and its related factors have gained importance in economic research. This paper analyzes the influence of household socioeconomic factors on the probability that children attend a public or private school at both the primary and secondary levels in Argentina. It also shows the composition of the primary and secondary school student body according to the educational level of the head of household and the social class to which the household belongs, using data from the Permanent Household Survey for Argentina. The results are in line with complementary findings in the literature.
\end{abstract}

Keywords: probability; school regime; primary education; secondary education; socioeconomic stratification; Argentina. 


\section{Introducción}

El sistema educativo argentino se caracteriza por ser heterogéneo en una multiplicidad de dimensiones, tanto las referidas a los establecimientos en sí —como, por ejemplo, el tipo de gestión, el ámbito donde se encuentra la escuela, rural o urbano, la idoneidad de sus docentes y autoridades - como a las de los estudiantes que asisten a ellas. Entre estas últimas se pueden mencionar, entre otras, a las características socioeconómicas de los hogares en donde estos viven. Dichos factores se asocian con heterogeneidades en el desarrollo cognitivo de los estudiantes, su desempeño educativo, las posibilidades de continuar con estudios superiores, el acceso al mercado formal de empleo y el perfil de ingresos futuros de los alumnos.

Cabe resaltar que existen diferencias en el desempeño educativo medio entre estudiantes en instituciones de gestión pública y privada. Tal como surge de los resultados de las pruebas Aprender 2017 (Ministerio de Educación de la República Argentina, 2017), los alumnos de escuelas privadas son quienes obtuvieron mejores resultados. A través de un análisis de clusters —en el cual el algoritmo agrupó a las escuelas según el desempeño promedio de los alumnos del último año de la escuela media en las evaluaciones de lengua y matemática - se identificaron como óptimos tres grupos de establecimientos que se denominaron: con rendimiento bajo, medio y alto. La proporción de escuelas privadas y públicas en cada uno de ellos es muy heterogénea. El $46 \%$ de los establecimientos privados registra alumnos con desempeño promedio elevado, $45 \%$ medio y $9 \%$ bajo; mientras que, en los establecimientos públicos, dichos valores son del 7\%, 38,5 $\%$ y $54,5 \%$, respectivamente. Esto motivó a estimar la probabilidad de que un estudiante asista a una institución de gestión pública dado el nivel educativo del jefe de hogar, condicional en los ingresos per cápita del hogar.

Dado que los alumnos que asisten a cada tipo de gestión escolar presentan diferentes características (Jaume, 2013), es relevante separar, en lo posible, la incidencia del nivel socioeconómico del hogar. Esto ha incentivado el estudio de las diferencias de rendimiento por nivel socioeconómico para cada tipo de gestión escolar. En este sentido, se encontró que el entorno socioeconómico correlaciona positivamente con el desempeño de los alumnos y este, a su vez, con el tipo de gestión de la escuela. Una serie de estudios ha hecho uso de los resultados de los exámenes estandarizados PISA en Argentina para mostrar que los alumnos que asisten a instituciones de gestión privada obtuvieron, en promedio, mejores resultados que sus pares 
pertenecientes a instituciones públicas (Albornoz et al., 2015), padres con mejor estatus ocupacional y mayor participación de alumnos en establecimientos educativos de gestión pública provenientes de hogares con padres de bajo nivel educativo (Krüger y Formichella, 2012; Krüger, 2019). No obstante, la evidencia acerca de si el tipo de gestión escolar tiene incidencia en el rendimiento escolar de los alumnos, aislando los efectos propios de su entorno socioeconómico, no es concluyente. Algunos estudios en Argentina encuentran efectos nulos (Cervini, 2006; Santos, 2007; Formichella, 2011 y Marchionni et al., 2012) y otros positivos (Llach y Cornejo, 2018; Fernández Aguerre, 2002; Llach, 2006; Fresoli et al., 2007) dependiendo de la especificación del modelo y de lo que se entienda por entorno socioeconómico.

En el presente análisis se observa que existe una mayor concentración de alumnos provenientes de hogares de "Clase Baja" en establecimientos públicos, al tiempo que son quienes demuestran tener peor desempeño académico. En los últimos años, la proporción de padres que envía a sus hijos a escuelas de gestión privada se ha incrementado en muchos países (Arcidiácono et al., 2014), entre los cuales se encuentra Argentina (Jaume, 2013), incluso entre los hogares de menores ingresos (Alderman et al., 2001). Es decir, la escuela pública, tan importante para la construcción del tejido social (Llach et al., 1999), está perdiendo participación.

En este contexto, el valor agregado de este trabajo es brindar evidencia acerca del cambio en la probabilidad de que un niño asista a una institución pública o privada en primaria o secundaria en función del nivel educativo del jefe de hogar, condicional en la posición del hogar en la distribución del ingreso. Es decir, se estudia aquí cuánto incide en la elección del tipo de gestión de la escuela el nivel educativo del padre y la situación económica, presentada en este artículo como la clase social a la que pertenece la familia en función de su ingreso per cápita familiar. El trabajo se estructura de la siguiente manera: la siguiente sección describe la fuente de datos y la metodología utilizada, luego se presentan los resultados de las predicciones y finalmente se presentan las conclusiones.

\section{Datos y metodología}

Para poder llevar a cabo el estudio, se construyó una base de datos original compuesta por todos los trimestres disponibles de la base correspondiente al cuestionario individual de la Encuesta Permanente de Hogares (EPH) correspondientes a los años 2016 a 2019. La EPH es una encuesta nacional, con periodicidad trimestral, representativa de la población urbana del país, que incluye preguntas relacionadas a las características demográficas, educativas, laborales y 
socioeconómicas de la población. Para el armado de la base se identificaron los hogares y dentro de estos a los estudiantes de nivel primario y secundario, junto con el jefe de hogar, sus características educativas y las características socioeconómicas del hogar. ${ }^{1}$

Se utilizó un modelo Probit para predecir la probabilidad de que un hijo asista a un colegio público o privado, condicional en el nivel educativo del jefe de hogar y controlando por la posición del hogar en la distribución del ingreso. Se definieron seis posibles niveles educativos del jefe de hogar: primario incompleto, primario completo, secundario incompleto, secundario completo, superior incompleto y superior completo. ${ }^{2}$ Para evitar que las dispersiones geográficas de los precios alteraran la posición relativa de un hogar en la distribución del ingreso nacional, se usaron las clasificaciones decílicas de las provincias. Siguiendo a Groisman (2016), se generaron tres clases sociales denominadas "Clase Baja", "Clase Media” y "Clase Alta”. La división estándar de las clases agrupa a los dos primeros deciles en la "Clase Baja"; a los deciles tres a ocho en "Clase Media" y los deciles nueve y diez en "Clase Alta".

En este estudio se adaptaron estas categorías a lo que se considera una estratificación más realista del caso argentino para los años 2016-2019. La tasa de pobreza de personas reportada por el Instituto Nacional de Estadísticas y Censos (INDEC) rondó el 30 \% para 2016, 27 \% para 2017, $30 \%$ para 2018 y $35 \%$ para $2019 .{ }^{3}$ En este análisis, la "Clase Baja" comprende hogares de los tres primeros deciles de ingreso; la "Clase Media" los hogares de los deciles cuatro al ocho y la "Clase Alta", los de los deciles nueve y diez. Con relación al nivel escolar al que asiste el hijo de cada tipo de hogar, cabe aclarar que en Argentina existen dos sistemas: algunas provincias cuentan con una primaria de siete años y una secundaria de cinco, mientras que en aquellas que han introducido la Educación General Básica (EGB) y el Polimodal, se incluyen los seis primeros años de EGB en nivel primario y los últimos tres años de EGB en nivel secundario.

\footnotetext{
${ }^{1}$ Dado que la encuesta releva durante cuatro ondas a cada hogar, se han eliminado todas las observaciones duplicadas.

${ }^{2}$ Aquellos jefes que carecían de cualquier tipo de instrucción fueron considerados como observaciones con primaria incompleta. A su vez, 284 hogares (0,25\% de la muestra) fueron eliminados del análisis porque registraban dos jefes. Este problema no surge de la existencia de dos hogares dentro de la misma vivienda, ya que en la base se cuenta con identificadores únicos al nivel del hogar.

3 La metodología utilizada por INDEC para calcular la línea de pobreza hace uso del ingreso total familiar para determinar si el hogar puede satisfacer sus necesidades alimentarias y no alimentarias. Se establece una canasta básica de alimentos (CBA) que luego se amplía con la inclusión de bienes y servicios no alimentarios (vestimenta, transporte, educación, salud, etc.) para así estimar el costo de la canasta básica total (CBT). El costo de la CBT es lo que se compara contra el ingreso total familiar para determinar la proporción de hogares y personas pobres.
} 
El modelo estimado para obtener las predicciones resulta ser un Probit del estilo

$$
\text { Institución }=\propto+\beta \text { Educ }+\gamma \text { Clase }+\varepsilon
$$

donde Institución es una variable binaria que vale 1 si el hijo asiste a una institución de gestión pública; Educ es un efecto fijo por nivel educativo del jefe de hogar y Clase, un efecto fijo por clase social que se generó, siendo $\varepsilon$ el término de error. ${ }^{4}$ En Educ se usó el nivel educativo "Primaria Incompleta" como nivel base y en Clase, la "Clase Baja".

\section{Resultados}

Tras estimar el modelo presentado en la sección anterior, se predijo Institución, de forma tal de contar con la probabilidad de elección del tipo de gestión de la escuela para cada nivel educativo del jefe y clase social del hogar. Las Figuras 1 y 2 muestran la proporción de niños en primarias/secundarias ${ }^{5}$ de gestión pública y privada, acompañada de la probabilidad condicional de asistir a una institución primaria/secundaria de gestión pública. Las Tablas 5 y 6 del Anexo cuentan con las estimaciones de las regresiones. Se observa que los resultados están en línea con otros hallazgos de la literatura. La probabilidad de asistir a una institución de gestión pública disminuye a medida que aumenta el nivel educativo del jefe de hogar y la posición del hogar en la distribución del ingreso.

Dentro de cada una de las clases sociales armadas, se observa que a medida que aumenta el nivel educativo del jefe de hogar, la probabilidad de que el hijo asista a una institución pública siempre disminuye. Cabe aclarar que las estimaciones realizadas muestran correlaciones y no causalidad. También se observa que la escuela pública cumple un rol importantísimo en términos de participación. El 68,5 \% del total de alumnos en primaria asiste a un establecimiento de gestión pública, del cual el 74 \% son alumnos que provienen de hogares de "Clase Baja”, mientras que el

\footnotetext{
${ }^{4}$ Para llevar las estimaciones de la muestra a la población se usó el peso PONDIH.

${ }^{5}$ Las proporciones fueron calculadas sobre el total de alumnos en cada nivel educativo, es decir, sobre la totalidad de alumnos en primaria o en secundaria, según corresponda.
} 
24\% proviene de hogares de "Clase Media" y apenas un 2\%, de hogares de "Clase Alta". Este hallazgo va en línea con lo encontrado en otros trabajos utilizando las pruebas PISA. ${ }^{6}$

Para el nivel secundario los valores aumentan al $73 \%$ del total de los alumnos, con $68,5 \%$ provenientes de hogares de "Clase Baja", 29,3\% provenientes de hogares de "Clase Media" y 2,2\%, de hogares de "Clase Alta". Resulta entonces que, al comparar las participaciones en primaria y secundaria de gestión pública, no solo se ve un aumento en el total, sino también un cambio en las decisiones de los jefes de hogares de "Clase Media" sobre la escolaridad de sus hijos. Por lo tanto, medido en términos de participación, la escuela secundaria pública funciona como un mejor integrador de alumnos de distintas clases sociales en relación con la escuela primaria pública. $^{7}$

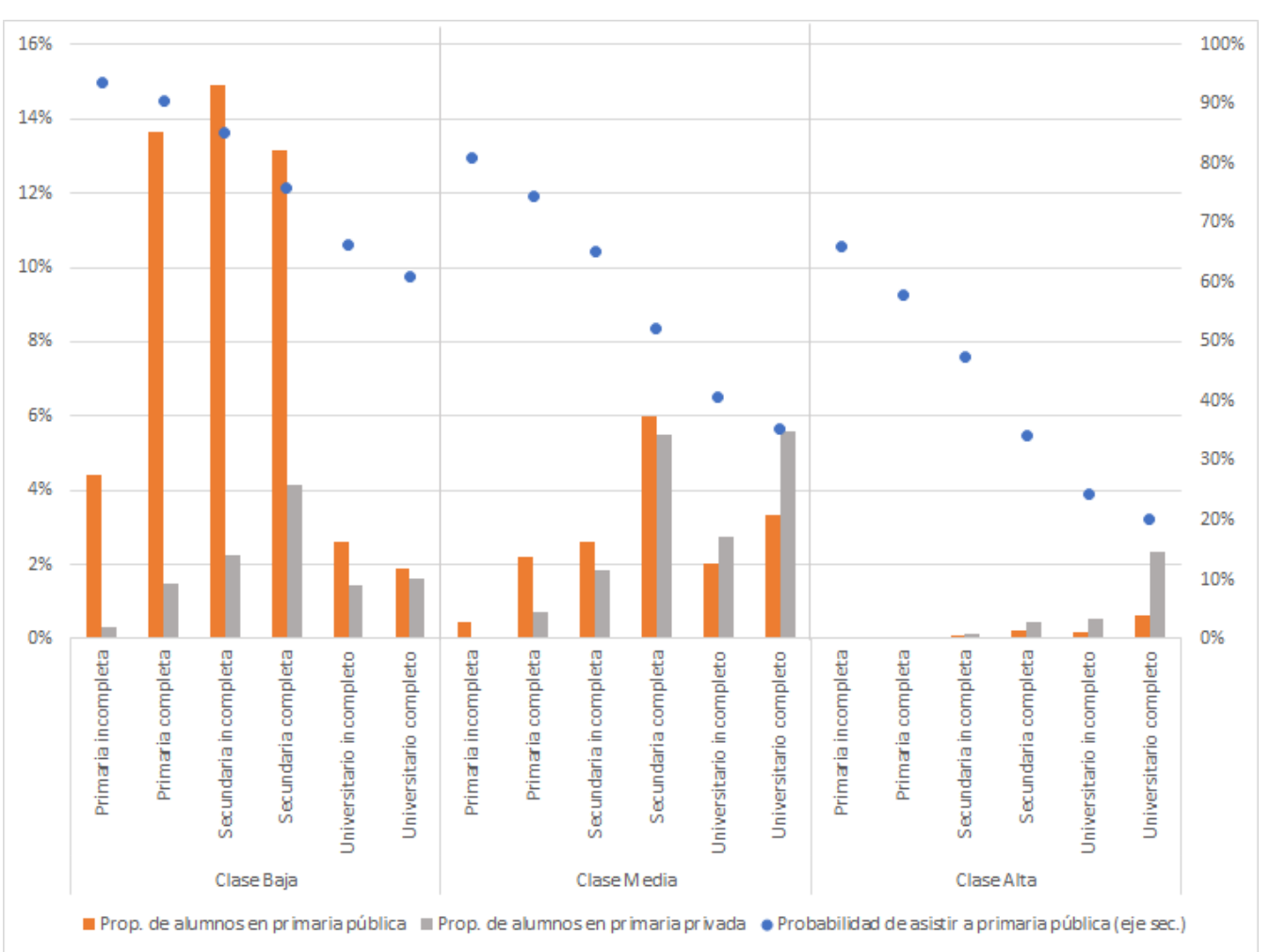

Figura 1. Probabilidad de asistir a establecimiento público en la escuela primaria y proporción de niños en escuela pública y privada condicional en la educación del jefe y la posición del hogar en la distribución del ingreso. Fuente: Elaboración propia en base a EPH 2016 a 2019 todos los trimestres disponibles.

${ }^{6}$ Estas proporciones no son robustas a la arbitrariedad de definir las clases sociales. Los resultados con la estratificación de clases que se adoptaron comparados con la propuesta por Groisman (2016) dan cuenta de ello en las Tablas 1 a 4 del Anexo.

${ }^{7} \mathrm{Si}$ se utilizara la estratificación tradicional de Groisman (2016), el total de las participaciones no se modifica, pero sí su composición. Se ve que la participación de alumnos de "Clase Baja” en la primaria pública es del $59 \%$ y el $39 \%$ son alumnos de "Clase Media" y el $2 \%$ de "Clase Alta". Para el nivel secundario, el 5 2\% de los alumnos son de "Clase Baja", el $46 \%$ de "Clase Media" y el $2 \%$ de "Clase Alta". 


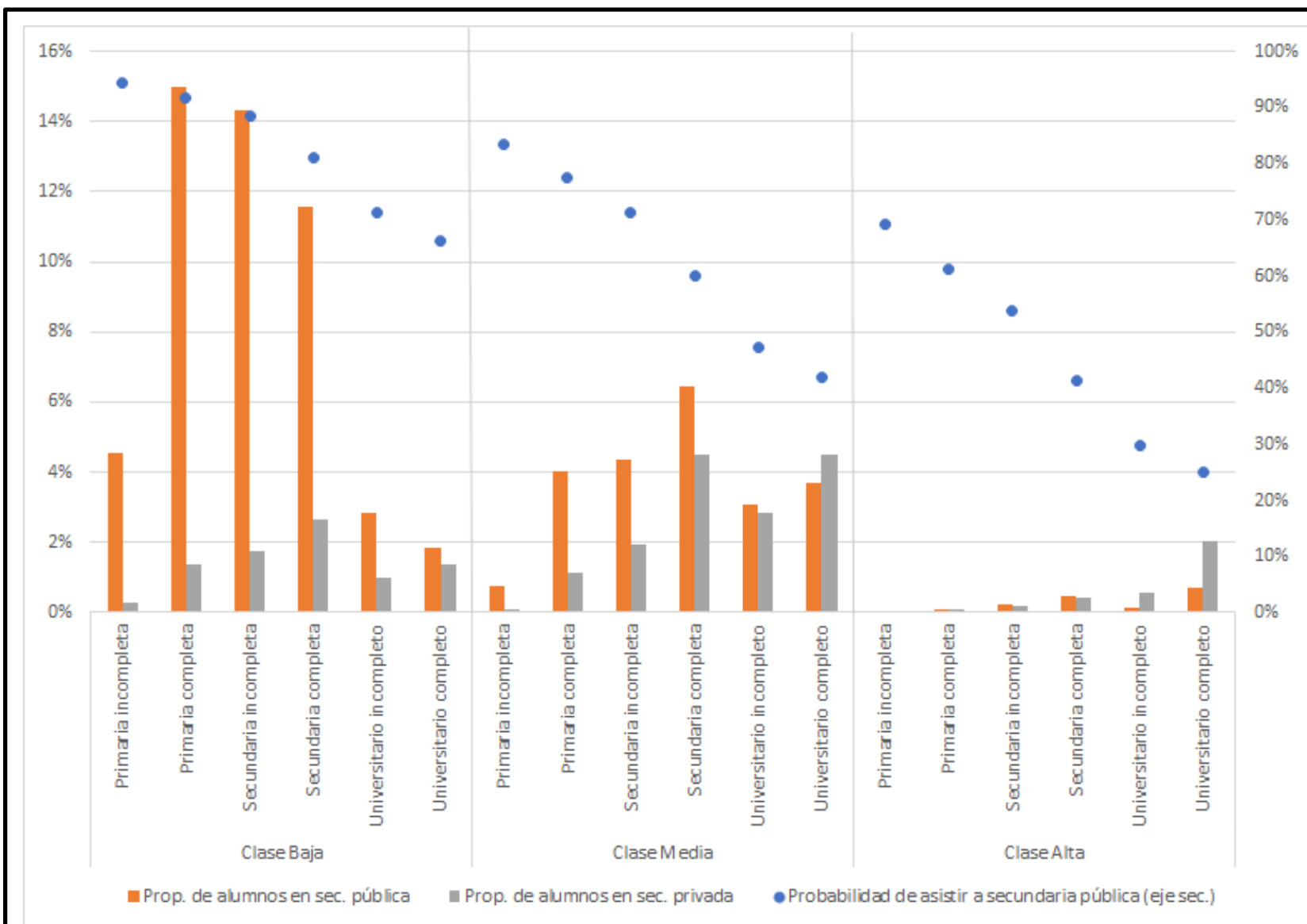

Figura 2. Probabilidad de asistir a establecimiento público en la escuela secundaria y proporción de niños en escuela pública y privada condicional en la educación del jefe y la posición del hogar en la distribución del ingreso. Fuente: Elaboración propia en base a EPH 2016 a 2019 todos los trimestres disponibles.

La Figura 3 muestra que, para un mismo nivel educativo del jefe y clase social del hogar, la probabilidad de que un hijo asista a primaria privada es, en promedio, mayor a que lo haga a secundaria privada. Esto lleva a creer que, en cierto modo, se revelan las preferencias de la sociedad argentina en estos términos. Los datos parecen indicar que, en promedio, los jefes de hogar, mayormente de los hogares de "Clase Media", le asocian a la educación primaria de gestión privada de sus hijos un beneficio marginal mayor al de la educación secundaria de igual gestión. Dar cuenta de por qué el fenómeno ocurre de este modo abre puertas a futuros trabajos de investigación. 


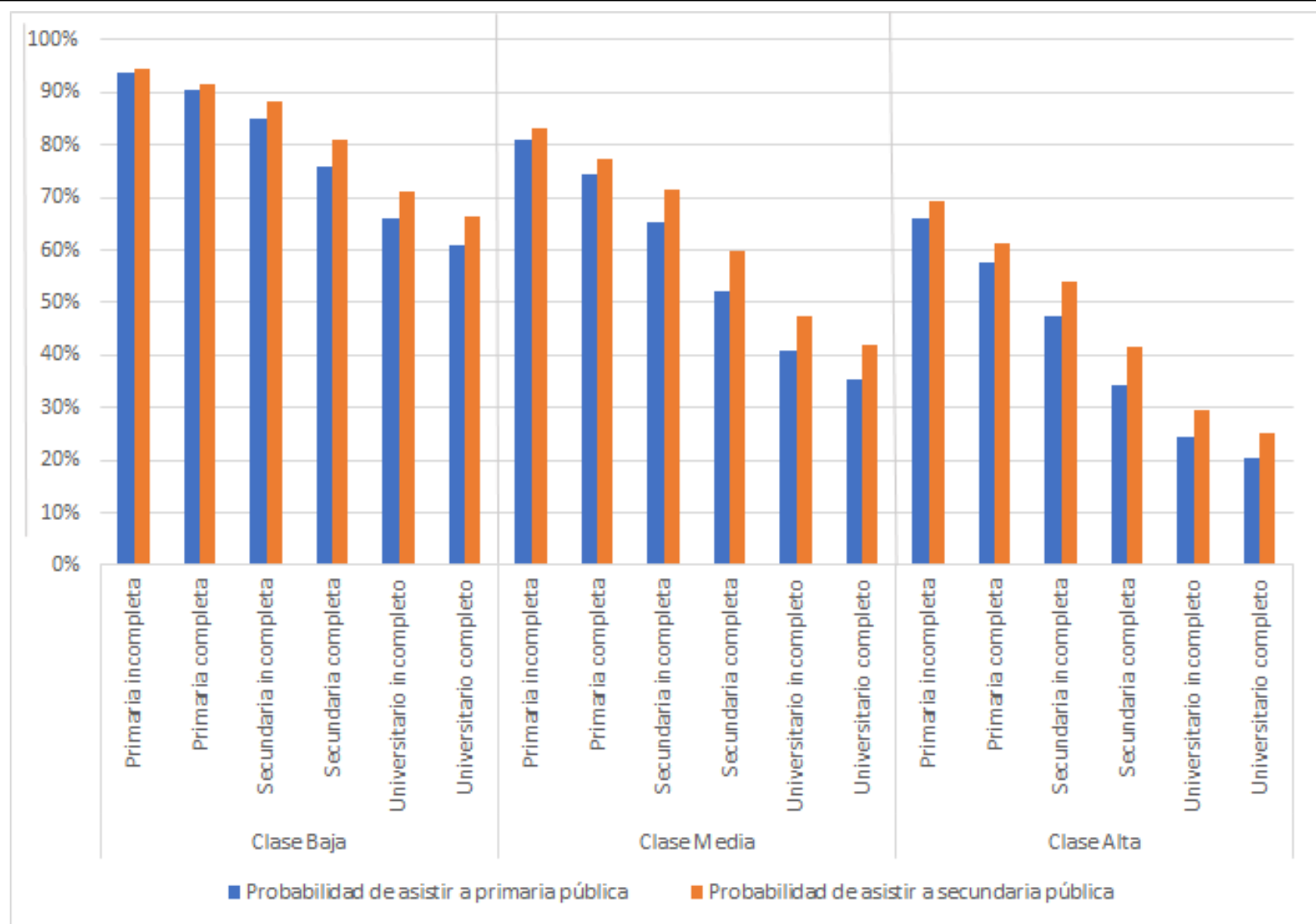

Figura 3. Probabilidades de asistir a primaria y secundaria pública condicional en la educación del jefe y en la posición del hogar en la distribución del ingreso. Fuente: Elaboración propia en base a EPH 2016 a 2019 todos los trimestres disponibles.

\section{Conclusiones}

Este trabajo intenta arrojar luz acerca de la importancia de los factores socioeconómicos y educativos en la elección del tipo de gestión de la escuela primaria y secundaria en Argentina. Sin duda, hay una multiplicidad de factores que no han podido ser incorporados, tales como los días de paro en las escuelas privadas y públicas (Jaume y Willén, 2019), la cercanía entre los hogares de los alumnos y las instituciones, que seguramente afectan tanto el rendimiento de los alumnos como la elección de los padres sobre el tipo de institución en la que se educarán sus hijos. No obstante, creemos que el hecho de poder separar los factores socioeconómicos y educativos de los hogares para estimar la probabilidad de que los hijos en edad escolar asistan a un tipo de establecimiento u otro, ayuda a entender un proceso que se viene observando: el aumento en la matrícula en los establecimientos privados. 
En este sentido, las conclusiones se pueden agrupar en tres:

i) A igualdad de nivel educativo del jefe de hogar, tener mayor capacidad económica permite que los padres opten por educar a sus hijos en escuelas privadas

ii) A igualdad de nivel socioeconómico del hogar, tener padres más educados eleva la probabilidad de que los hijos asistan a establecimientos de gestión privada. Esto se verifica para las tres clases sociales armadas y está en línea con resultados encontrados en la literatura (Krüger y Formichella, 2012).

iii) A igualdad de nivel educativo de los jefes y socioeconómico del hogar, la probabilidad de asistir a un establecimiento de gestión pública es mayor para el nivel secundario que para el primario.

En un futuro próximo será interesante evaluar si se modifican, y de qué manera, las asignaciones de alumnos entre escuelas privadas y públicas. La irrupción de la pandemia provocada por la COVID-19 trajo consigo no solo una crisis sanitaria, sino también económica y social que afectará la posibilidad de afrontar los costos de una educación privada. Posiblemente esto se verá reflejado en un aumento en la proporción de estudiantes en escuelas de gestión pública en ambos niveles, lo cual impondrá desafíos a la capacidad de la educación de gestión pública de poder absorber el shock de demanda.

Como las escuelas han dejado de ser un espacio de encuentro personal para convertirse en espacios de encuentro "virtual", las exigencias sobre la capacidad instalada de los establecimientos públicos podría no ser un problema. De todos modos, las exigencias sobre los recursos docentes se harían sentir, incrementándose las erogaciones del sector público. Este shock actuará como un experimento natural que impactará sobre la capacidad económica de los hogares y no sobre el nivel educativo de los jefes de hogar y permitirá, en gran medida, aislar los componentes económicos de los años de educación de los jefes de hogar. 


\section{Referencias}

Albornoz, F., Furman, M., Podestá, M. E., Razquin, P., \& Warnes, P. E. (2016). Diferencias educativas entre escuelas privadas y públicas en Argentina. Desarrollo económico, 56(218), 3-31.

Alderman, H., Orazem, P. F., \& Paterno, E. M. (2001). School quality, school cost, and the public/private school choices of low-income households in Pakistan. Journal of Human resources, 304-326.

Arcidiácono, M., Cruces, G., Gasparini, L., Jaume, D., Serio, M., \& Vázquez, E. (2014). La segregación escolar público-privada en América Latina. Cepal.

Cervini, R. (2006). Los efectos de la escuela y del aula sobre el logro en matemáticas y en lengua de la educación secundaria. Un modelo multinivel. Perfiles educativos, vol. 28, N 112, México, D.F., Universidad Nacional Autonoma de Mexico.

Fernández Aguerre, T. (2002). Determinantes sociales e institucionales de la desigualdad educativa en sexto año de educación primaria de Argentina y Uruguay, 1999. Una aproximación mediante un modelo de regresión logística, Revista mexicana de investigación educativa, vol. 7, No 16, México, D.F., Consejo Mexicano de Investigación Educativa.

Formichella, M. (2011). ¿Se debe el mayor rendimiento de las escuelas de gestión privada en la Argentina al tipo de administración? Revista de la CEPAL, núm. 105, pp. 151-166.

Fresoli, D., V. Herrero, R. Giuliodori y H. Gertel (2007). Incidencia de la gestión sobre el rendimiento escolar en la escuela argentina. El mensaje de las pruebas internacionales y nacionales. Anales de la Asociación Argentina de Economía Política.

Groisman, F. (2016). Estructura Social e Informalidad Laboral en Argentina. EUDEBA.

Jaume, D. (2013). Un estudio sobre el incremento de la segregación escolar en Argentina. Documentos de Trabajo del CEDLAS.

Jaume, D., \& Willén, A. (2019). The long-run effects of teacher strikes: evidence from Argentina. Journal of Labor Economics, 37(4), 1097-1139.

Krüger, N. (2019). La segregación por nivel socioeconómico como dimensión de la exclusión educativa: 15 años de evolución en América Latina. Archivos Analíticos de Políticas Educativas, 27(8).

Krüger, N., \& Formichella, M. M. (2012). Escuela pública y privada en Argentina: una comparación de las condiciones de escolarización en el nivel medio. Perspectivas, 6(1), 113-144.

Llach, J. (2006). El desafío de la equidad educativa. Diagnóstico y propuestas. Buenos Aires: Granica.

Llach, J. y Cornejo, M. (2018), Factores condicionantes de los aprendizajes. Primaria y Secundaria, Serie de Investigaciones $\mathrm{N}^{\circ} 3$, Ministerio de Educación de la Nación.

Llach, J., Montoya, S. y Roldán, F. (1999). Educación para Todos. Instituto de Estudios de la Realidad Argentina y Latinoamericana (IERAL). Buenos Aires.

Marchionni, M., Vazquez, E., y Pinto, F. (2012). Desigualdad educativa en la Argentina. Análisis en base a los datos PISA 2009 [Education Inequality in Argentina. An analysis based on PISA 2009 data] (No. 56420). University Library of Munich, Germany. 
Ministerio de Educación de la República Argentina. (2017). Pruebas Aprender 2017. https://www.argentina.gob.ar/educacion/aprender2017

Santos, M. (2007), Quality of education in Argentina: determinants and distribution using pisa 2000 test scores, Wellbeing and Social Policy, vol. 3, No 1, Mexico, D.F., Conferencia Interamericana de Seguridad Social/Universidad Iberoamericana.

Código JEL: I24

\section{Contribución autoral}

a) Concepción y diseño del trabajo; b) Adquisición de datos; c) Análisis e interpretación de datos; d) Redacción del manuscrito; e) revisión crítica del manuscrito.

J. S. ha contribuido en a, b, c, d, e; C. A. en a, b, c, d, e.

\section{Editora científica responsable}

Mag. Florencia de León 


\section{ANEXO}

Tabla 1

Probabilidad de asistir a una primaria de gestión pública en Argentina (Groisman ajustado*)

\begin{tabular}{ccccccc}
\hline $\begin{array}{c}\text { Clase } \\
\text { social }\end{array}$ & $\begin{array}{c}\text { Primario } \\
\text { incompleto }\end{array}$ & $\begin{array}{c}\text { Primario } \\
\text { completo }\end{array}$ & $\begin{array}{c}\text { Secundario } \\
\text { incompleto }\end{array}$ & $\begin{array}{c}\text { Secundario } \\
\text { completo }\end{array}$ & $\begin{array}{c}\text { Universitario } \\
\text { incompleto }\end{array}$ & $\begin{array}{c}\text { Universitario } \\
\text { completo }\end{array}$ \\
\hline $\begin{array}{c}\text { Clase } \\
\text { Baja }\end{array}$ & $93.63 \%$ & $90.42 \%$ & $85.17 \%$ & $75.98 \%$ & $66.18 \%$ & $60.91 \%$ \\
$\begin{array}{c}\text { Clase } \\
\text { Media }\end{array}$ & $80.90 \%$ & $74.39 \%$ & $65.30 \%$ & $52.21 \%$ & $40.79 \%$ & $35.44 \%$ \\
$\begin{array}{c}\text { Clase } \\
\text { Alta }\end{array}$ & $66.09 \%$ & $57.77 \%$ & $47.37 \%$ & $34.31 \%$ & $24.43 \%$ & $20.25 \%$ \\
\hline
\end{tabular}

*Las clases sociales se definen de la siguiente manera: "Clase Baja" deciles 1, 2 y 3; "Clase Media” deciles 4,5,6,7,8; "Clase Alta" deciles 9 y 10. El modelo se estima con 23.272 observaciones. En la "Clase Baja” contamos con 14.462 observaciones, 7.722 en la "Clase Media" y 1.088 en la "Clase Alta".

Tabla 2

Probabilidad de asistir a una secundaria de gestión pública en Argentina (Groisman ajustado*)

\begin{tabular}{ccccccc}
\hline $\begin{array}{c}\text { Clase } \\
\text { social }\end{array}$ & $\begin{array}{c}\text { Primario } \\
\text { incompleto }\end{array}$ & $\begin{array}{c}\text { Primario } \\
\text { completo }\end{array}$ & $\begin{array}{c}\text { Secundario } \\
\text { incompleto }\end{array}$ & $\begin{array}{c}\text { Secundario } \\
\text { completo }\end{array}$ & $\begin{array}{c}\text { Universitario } \\
\text { incompleto }\end{array}$ & $\begin{array}{c}\text { Universitario } \\
\text { completo }\end{array}$ \\
\hline $\begin{array}{c}\text { Clase } \\
\text { Baja }\end{array}$ & $94.44 \%$ & $91.57 \%$ & $88.31 \%$ & $80.94 \%$ & $71.16 \%$ & $66.36 \%$ \\
$\begin{array}{c}\text { Clase } \\
\text { Media }\end{array}$ & $83.34 \%$ & $77.39 \%$ & $71.40 \%$ & $59.89 \%$ & $47.33 \%$ & $41.96 \%$ \\
$\begin{array}{c}\text { Clase } \\
\text { Alta }\end{array}$ & $69.18 \%$ & $61.21 \%$ & $53.91 \%$ & $41.43 \%$ & $29.67 \%$ & $25.15 \%$ \\
\hline
\end{tabular}

*Las clases sociales se definen de la siguiente manera: "Clase Baja” deciles 1, 2 y 3; "Clase Media" deciles 4,5,6,7,8; "Clase Alta" deciles 9 y 10. El modelo se estima con 23.883 observaciones. En la "Clase Baja" contamos con 14.037 observaciones, 8.643 en la "Clase Media" y 1.203 en la "Clase Alta". 


\section{Tabla 3}

Probabilidad de asistir a una primaria de gestión pública en Argentina (Groisman tradicional**)

\begin{tabular}{ccccccc}
\hline $\begin{array}{c}\text { Clase } \\
\text { social }\end{array}$ & $\begin{array}{c}\text { Primario } \\
\text { incompleto }\end{array}$ & $\begin{array}{c}\text { Primario } \\
\text { completo }\end{array}$ & $\begin{array}{c}\text { Secundario } \\
\text { incompleto }\end{array}$ & $\begin{array}{c}\text { Secundario } \\
\text { completo }\end{array}$ & $\begin{array}{c}\text { Universitario } \\
\text { incompleto }\end{array}$ & $\begin{array}{c}\text { Universitario } \\
\text { completo }\end{array}$ \\
\hline $\begin{array}{c}\text { Clase } \\
\text { Baja }\end{array}$ & $95.09 \%$ & $92.49 \%$ & $87.69 \%$ & $79.49 \%$ & $69.96 \%$ & $64.44 \%$ \\
$\begin{array}{c}\text { Clase } \\
\text { Media }\end{array}$ & $83.93 \%$ & $78.14 \%$ & $69.07 \%$ & $56.42 \%$ & $44.49 \%$ & $38.53 \%$ \\
$\begin{array}{c}\text { Clase } \\
\text { Alta }\end{array}$ & $67.14 \%$ & $59.06 \%$ & $48.00 \%$ & $34.97 \%$ & $24.62 \%$ & $20.06 \%$ \\
\hline
\end{tabular}

**Las clases sociales se definen de la siguiente manera: "Clase Baja" deciles 1 y 2; "Clase Media" deciles 3,4,5,6,7,8; "Clase Alta" deciles 9 y 10. El modelo se estima con 23.272 observaciones. En la "Clase Baja” contamos con 11.033 observaciones, 11.151 en la "Clase Media" y 1.088 en la "Clase Alta".

Tabla 4 Probabilidad de asistir a una secundaria de gestión pública en Argentina (Groisman tradicional**)

\begin{tabular}{ccccccc}
\hline $\begin{array}{c}\text { Clase } \\
\text { social }\end{array}$ & $\begin{array}{c}\text { Primario } \\
\text { incompleto }\end{array}$ & $\begin{array}{c}\text { Primario } \\
\text { completo }\end{array}$ & $\begin{array}{c}\text { Secundario } \\
\text { incompleto }\end{array}$ & $\begin{array}{c}\text { Secundario } \\
\text { completo }\end{array}$ & $\begin{array}{c}\text { Universitario } \\
\text { incompleto }\end{array}$ & $\begin{array}{c}\text { Universitario } \\
\text { completo }\end{array}$ \\
\hline $\begin{array}{c}\text { Clase } \\
\text { Baja }\end{array}$ & $95.49 \%$ & $93.07 \%$ & $89.92 \%$ & $83.04 \%$ & $73.34 \%$ & $67.80 \%$ \\
$\begin{array}{c}\text { Clase } \\
\text { Media }\end{array}$ & $86.65 \%$ & $81.51 \%$ & $75.58 \%$ & $64.49 \%$ & $51.56 \%$ & $45.15 \%$ \\
$\begin{array}{c}\text { Clase } \\
\text { Alta }\end{array}$ & $70.73 \%$ & $63.02 \%$ & $55.10 \%$ & $42.35 \%$ & $29.97 \%$ & $24.62 \%$ \\
\hline
\end{tabular}

**Las clases sociales se definen de la siguiente manera: "Clase Baja" deciles 1 y 2; "Clase Media" deciles 3,4,5,6,7,8; "Clase Alta" deciles 9 y 10. El modelo se estima con 23.883 observaciones. En la "Clase Baja" contamos con 10.384 observaciones, 12.296 en la "Clase Media" y 1.203 en la "Clase Alta". 


\section{Tabla 5}

Estimaciones del modelo probit para alumnos en primaria

Educación del jefe y clase social

Primario completo $\quad-0.219^{*}$

$(0.120)$

Secundario incompleto

$-0.481 * * *$

$(0.117)$

Secundario completo

$-0.819 * * *$

$(0.114)$

Universitario incompleto

$-1.107 * * *$

$(0.121)$

Universitario completo

$-1.248^{* * *}$

(0.118)

Clase Media

$-0.650 * * *$

(0.0359)

Clase Alta

$-1.110^{* * *}$

(0.0767)

Constante

$1.525^{* * * *}$

$(0.111)$

Observaciones

23,272

Errores estándar robustos entre paréntesis $* * * \mathrm{p}<0.01, * * \mathrm{p}<0.05, * \mathrm{p}<0.1$ 


\section{Tabla 6}

Estimaciones del modelo probit para alumnos en secundaria

\section{(1)}

Educación del jefe y clase social

\begin{tabular}{lc}
\hline Primario completo & $-0.216^{*}$ \\
& $(0.112)$
\end{tabular}

Secundario incompleto

$-0.403 * * *$

$(0.110)$

Secundario completo

$-0.717 * * *$

$(0.108)$

Universitario incompleto

$-1.035^{* * *}$

(0.114)

Universitario completo

$-1.171 * * *$

(0.112)

Clase media

$-0.625 * * *$

$(0.0357)$

Clase alta

$-1.092 * * *$

$(0.0728)$

Constante

$1.593 * * *$

(0.104)

Observaciones

23,883

\section{Errores estándar robustos entre paréntesis $* * * \mathrm{p}<0.01, * * \mathrm{p}<0.05, * \mathrm{p}<0.1$}

